\title{
Адсорбция атомов II и VI групп на политипах карбида кремния
}

\author{
(C) С.Ю. Давыдов ${ }^{1}$, О.В. Посредник ${ }^{2}$ \\ ${ }^{1}$ Физико-технический институт им. А.Ф. Иоффе Российской академии наук, \\ 194021 Санкт-Петербург, Россия \\ ${ }^{2}$ Санкт-Петербургский государственный электротехнический университет (ЛЭТИ), \\ 197376 Санкт-Петербург, Россия \\ E-mail: Sergei_Davydov@mail.ru
}

Поступила в Редакцию 30 октября 2020 г. В окончательной редакции 14 ноября 2020 г. Принята к публикации 14 декабря 2020 г.

В рамках модели Халдейна-Андерсона получены оценки перехода заряда и энергии адсорбции атомов щелочноземельных металлов и атомов подгруппы кислорода на C- и $\mathrm{Si}$-гранях $3 C$ - и $4 H$-политипов $\mathrm{SiC}$. Выявлены вклады зонных и локальных состояний в переход заряда между адатомами и подложками, металлической и ионной составляющих энергии адсорбции.

Ключевые слова: адатом, подложка, переход заряда, энергия адсорбции.

DOI: $10.21883 /$ FTP.2021.04.50733.9548

\section{1. Введение и модель адсорбции}

Карбид кремния, характеризуемый большим количеством политипов с существенно различающимися ширинами запрещенных зон и высокой стойкостью к агрессивным средам, по-прежнему привлекает пристальное внимание исследователей [1].

Настоящей публикацией мы продолжаем систематические исследования адсорбционной способности $\mathrm{SiC}$, начатое в работе [2], где мы рассмотрели адсорбцию атомов I и VII групп на C- и Si-гранях 3C-, 6H- и $4 H$-политипах карбида кремния, причем основное внимание уделялось оценкам перехода заряда и энергии адсорбции. При этом использовался модельный подход к проблеме адсорбции, изложенный в $[3,4]$. В настоящей работе мы рассмотрим адсорбцию на $3 C-\mathrm{SiC}$ и $4 H-\mathrm{SiC}$ атомов главной подгруппы (или подгруппы бериллия) II группы и подгруппы кислорода VI группы, следуя схеме работы [2], где можно найти подробное обоснование всех математических выкладок.

В рамках модельного подхода [2] плотность состояний $\rho_{a}(\omega)$ на адатоме может быть представлена в виде контура Лорентца вида

$$
\rho_{a}(\omega)=\frac{1}{\pi} \frac{\Gamma(\omega)}{\left[\omega-\varepsilon_{a}-\Lambda(\omega)\right]^{2}+\Gamma^{2}(\omega)},
$$

где $\omega-$ энергетическая переменная, $\varepsilon_{a}-$ энергия уровня адсорбируемого атома, $\Gamma(\omega)=\pi V^{2} \rho_{\text {sub }}(\omega)-$ функция уширения квазиуровня $\left(\rho_{\mathrm{sub}}(\omega)-\right.$ плотность состояний подложки, $V$ - матричный элемент взаимодействия адатом-подложка) и $\Lambda(\omega)$ - функция сдвига квазиуровня (гильбертова трансформанта функции $\Gamma(\omega))$. Для описания плотности состояний карбида кремния воспользуемся моделью Халдейна-Андерсона [2-4]: $\rho_{\text {sub }}(\omega)=\rho_{s}$ для $\left|\omega-E_{0}\right| \geq E_{g} / 2$ и $\rho_{\text {sub }}(\omega)=0$ при $\left|\omega-E_{0}\right|<E_{g} / 2$, где $E_{0}=\chi+E_{g} / 2-$ энергия центра запрещенной зоны относительно вакуума, $E_{g}$ и $\chi-$ ширина запрещенной зоны и электронное сродство политипа карбида кремния. Полагая $E_{0}=0$, получим

$$
\Lambda(\omega)=(\Gamma / \pi) \ln \left|\left(\omega-E_{g} / 2\right) /\left(\omega+E_{g} / 2\right)\right|,
$$

где $\Gamma \equiv \pi V^{2} \rho_{s}=$ const. Для $3 C$-, $6 H$ - и $4 H$-политипов карбида кремния соответственно имеем $E_{g}=2.40,3.00$ и 3.23 эВ, $\chi=4.00,3.45$ и 3.17 эВ [5]. Здесь мы ограничимся случаями $\mathrm{C}$ - и $\mathrm{Si}-г$ раней $3 C$ - и $4 H-\mathrm{SiC}$, так как именно эти подложки обладают максимально различающимися значениями $E_{g}$ и $\chi$.

\section{2. Расчеты}

\section{1. Оценки параметров модели}

Перейдем к оценкам параметров модели и начнем c адатомов II группы. Энергия уровня адатома $\varepsilon_{a}^{0}=-I+e^{2} / 4 d \quad$ относительно вакуума $\quad$ и $\varepsilon_{a}=\varepsilon_{a}^{0}$ $+\left(\chi+E_{g} / 2\right)$ относительно центра запрещенной зоны политипа $\mathrm{SiC}$, где $I-$ энергия ионизации [6], $d-$ длина адсорбционной связи, $e-$ элементарный заряд. Полагаем $d(\mathrm{M}-\mathrm{C}, \mathrm{Si})=r_{a}(\mathrm{C}, \mathrm{Si})+r_{a}(\mathrm{M})$, где $r_{a}(\mathrm{C}, \mathrm{Si})$ - атомные радиусы углерода или кремния, $r_{a}(\mathrm{M})$ - атомный радиус щелочноземельного атома [6]. В работе [2] для адсорбции атома лития на С-грани мы положили $\Gamma(\mathrm{Li})=1$ эВ. Значения $\Gamma_{a}$ для рассматриваемых в настоящей работе адатомов будем определять исходя из того, что $\Gamma \propto d^{-4} \quad[1] . \quad$ Тогда $\quad \Gamma(\mathrm{M}-\mathrm{C}, \mathrm{Si})=\gamma_{\mathrm{C}, \mathrm{Si}} \Gamma(\mathrm{Li}-\mathrm{C}, \mathrm{Si})$ и $\quad \gamma_{\mathrm{C}, \mathrm{Si}}=[d(\mathrm{Li}-\mathrm{C}) / d(\mathrm{M}-\mathrm{C}, \mathrm{Si})]^{4}$. Значения $d$, $\Gamma(\mathrm{M}-\mathrm{C}, \mathrm{Si})$ и $\varepsilon_{a}$ представлены в табл. 1.

Обратимся теперь к оценкам параметров для адатомов VI группы. Энергия уровня адатома равна $\varepsilon_{a}^{0}=-A-e^{2} / 4 d$ относительно вакуума и $\varepsilon_{a}=\varepsilon_{a}^{0}+\left(\chi+E_{g} / 2\right)$ относительно центра запрещенной зоны политипа $\mathrm{SiC}$, где $A-$ сродство атома к электрону [6]. Полагаем $d(\mathrm{X}-\mathrm{C}, \mathrm{Si})=r_{a}(\mathrm{C}, \mathrm{Si})+r_{a}(\mathrm{X})$ где 
Таблица 1. Параметры адатомов II группы (длины связей в $\AA$, энергетические величины - в эВ)

\begin{tabular}{c|c|c|c|c|c}
\hline $\mathrm{M}$ & $\mathrm{Be}$ & $\mathrm{Mg}$ & $\mathrm{Ca}$ & $\mathrm{Sr}$ & $\mathrm{Ba}$ \\
\hline$d_{\mathrm{M}-\mathrm{C}}$ & 1.90 & 2.37 & 2.74 & 2.92 & 3.01 \\
$d_{\mathrm{M}-\mathrm{Si}}$ & 2.31 & 2.78 & 3.15 & 3.33 & 3.42 \\
$\Gamma(\mathrm{M}-\mathrm{C})$ & 2.30 & 0.95 & 0.53 & 0.41 & 0.365 \\
$\Gamma(\mathrm{M}-\mathrm{Si})$ & 1.02 & 0.49 & 0.295 & 0.24 & 0.21 \\
$3 C-\mathrm{SiC}$ & & & & & \\
$\varepsilon_{a}(\mathrm{M}-\mathrm{C})$ & -2.23 & -0.92 & 0.40 & 0.74 & 1.19 \\
$\varepsilon(\mathrm{M}-\mathrm{Si})$ & -2.56 & -1.15 & 0.23 & 0.69 & 1.04 \\
$4 H-\mathrm{SiC}$ & & & & & \\
$\varepsilon_{a}(\mathrm{M}-\mathrm{C})$ & -2.65 & -1.34 & -0.02 & 0.33 & 0.78 \\
$\varepsilon(\mathrm{M}-\mathrm{Si})$ & -2.98 & -1.57 & -0.19 & 0.28 & 0.65 \\
$3 C-\mathrm{SiC}$ & & & & & \\
$\omega_{l}(\mathrm{M}-\mathrm{C})$ & -0.88 & -0.60 & 0.30 & 0.60 & 0.95 \\
$\omega_{l}(\mathrm{M}-\mathrm{Si})$ & -1.17 & -0.87 & 0.20 & 0.61 & 0.91 \\
$4 H-\mathrm{SiC}$ & & & & & \\
$\omega_{l}(\mathrm{M}-\mathrm{C})$ & -1.22 & -0.94 & -0.01 & 0.28 & 0.67 \\
$\omega_{l}(\mathrm{M}-\mathrm{Si})$ & -1.57 & -1.25 & -0.17 & 0.25 & 0.56
\end{tabular}

Таблица 2. Параметры адатомов VI группы (длины связей в $\AA$, энергетические величины - в эВ)

\begin{tabular}{c|c|c|c|c}
\hline $\mathrm{TX}$ & $\mathrm{O}$ & $\mathrm{S}$ & $\mathrm{Se}$ & $\mathrm{Te}$ \\
\hline$d_{\mathrm{X}-\mathrm{C}}$ & 1.51 & 1.81 & 2.37 & 2.47 \\
$d_{\mathrm{X}-\mathrm{Si}}$ & 1.92 & 2.22 & 2.78 & 2.88 \\
$\Gamma(\mathrm{X}-\mathrm{C})$ & 5.77 & 2.79 & 0.95 & 0.81 \\
$\Gamma(\mathrm{X}-\mathrm{Si})$ & 2.14 & 1.20 & 0.49 & 0.42 \\
$3 C-\mathrm{SiC}$ & & & & \\
$\varepsilon_{a}(\mathrm{X}-\mathrm{C})$ & 1.36 & 1.13 & 1.66 & 1.77 \\
$\varepsilon_{a}(\mathrm{X}-\mathrm{Si})$ & 1.85 & 1.50 & 1.89 & 1.98 \\
$4 H-\mathrm{SiC}$ & & & & \\
$\varepsilon_{a}(\mathrm{X}-\mathrm{C})$ & 0.95 & 0.72 & 1.25 & 1.34 \\
$\varepsilon_{a}(\mathrm{X}-\mathrm{Si})$ & 1.44 & 1.09 & 1.48 & 1.57 \\
$3 C-\mathrm{SiC}$ & & & & \\
$\omega_{l}(\mathrm{X}-\mathrm{C})$ & 0.33 & 0.44 & 0.97 & 1.05 \\
$\omega_{l}(\mathrm{X}-\mathrm{Si})$ & 0.75 & 0.83 & 1.18 & 1.19 \\
$4 H-\mathrm{SiC}$ & & & & \\
$\omega_{l}(\mathrm{X}-\mathrm{C})$ & 0.29 & 0.53 & 0.88 & 0.97 \\
$\omega_{l}(\mathrm{X}-\mathrm{Si})$ & 0.75 & 0.72 & 1.18 & 1.28
\end{tabular}

$\mathrm{X}=\mathrm{O}, \mathrm{S}, \mathrm{Se}, \mathrm{Te}$ (величины $r_{a}(\mathrm{X})$ брались из [6]). Далее, как показано в [2], $\Gamma(\mathrm{X}-\mathrm{C}, \mathrm{Si})=\gamma^{\prime} \gamma \Gamma(\mathrm{Li}-\mathrm{C}, \mathrm{Si})$, где $\gamma^{\prime}=0.97$. Значения $d, \Gamma(\mathrm{X}-\mathrm{C}, \mathrm{Si})$ и $\varepsilon_{a}$ представлены в табл. 2.

\section{2. Переход заряда}

Рассмотрим переход заряда между адатомом и подложкой при нулевой температуре, для чего рассчитаем
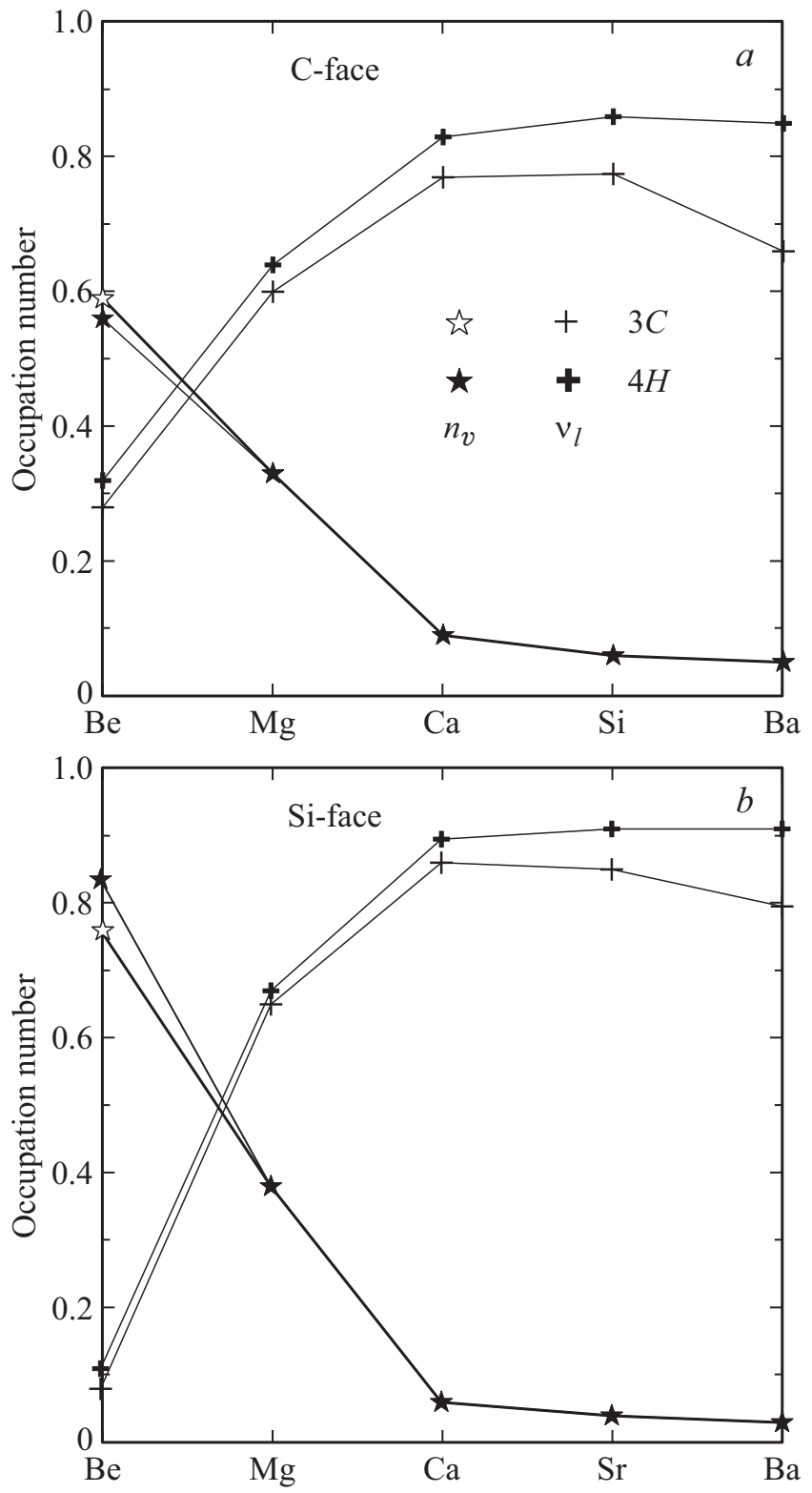

Рис. 1. Адатомы II группы: числа заполнения зонных $n_{v}$ (звездочки) и локальных $v_{l}$ (крестики) состояний на С- и Si-гранях (соответственно $a$ и $b$ ) политипов $3 C$ (светлые символы) и $4 H$ (темные символы).

числа заполнения $n=\int_{-\infty}^{E_{\mathrm{F}}} \rho_{a}(\omega) d \omega$, где $E_{\mathrm{F}}-$ уровень Ферми. Положим $n=n_{v}+n_{l}$, где $n_{v}$ и $n_{l}$ представляют соответственно вклады валентной зоны и локального уровня, находящегося в запрещенной зоне. Согласно [7], вклад валентной зоны

$$
n_{v}=\frac{1}{\pi} \operatorname{arcctg} \frac{\varepsilon_{a}+R}{\Gamma}, \quad R=\frac{E_{g}}{2} \sqrt{1+\frac{4 \Gamma}{\pi E_{g}}} .
$$

Положение локального уровня $\omega_{l}$ определяется из решения уравнения

$$
\omega-\varepsilon_{a}-\Lambda(\omega)=0
$$



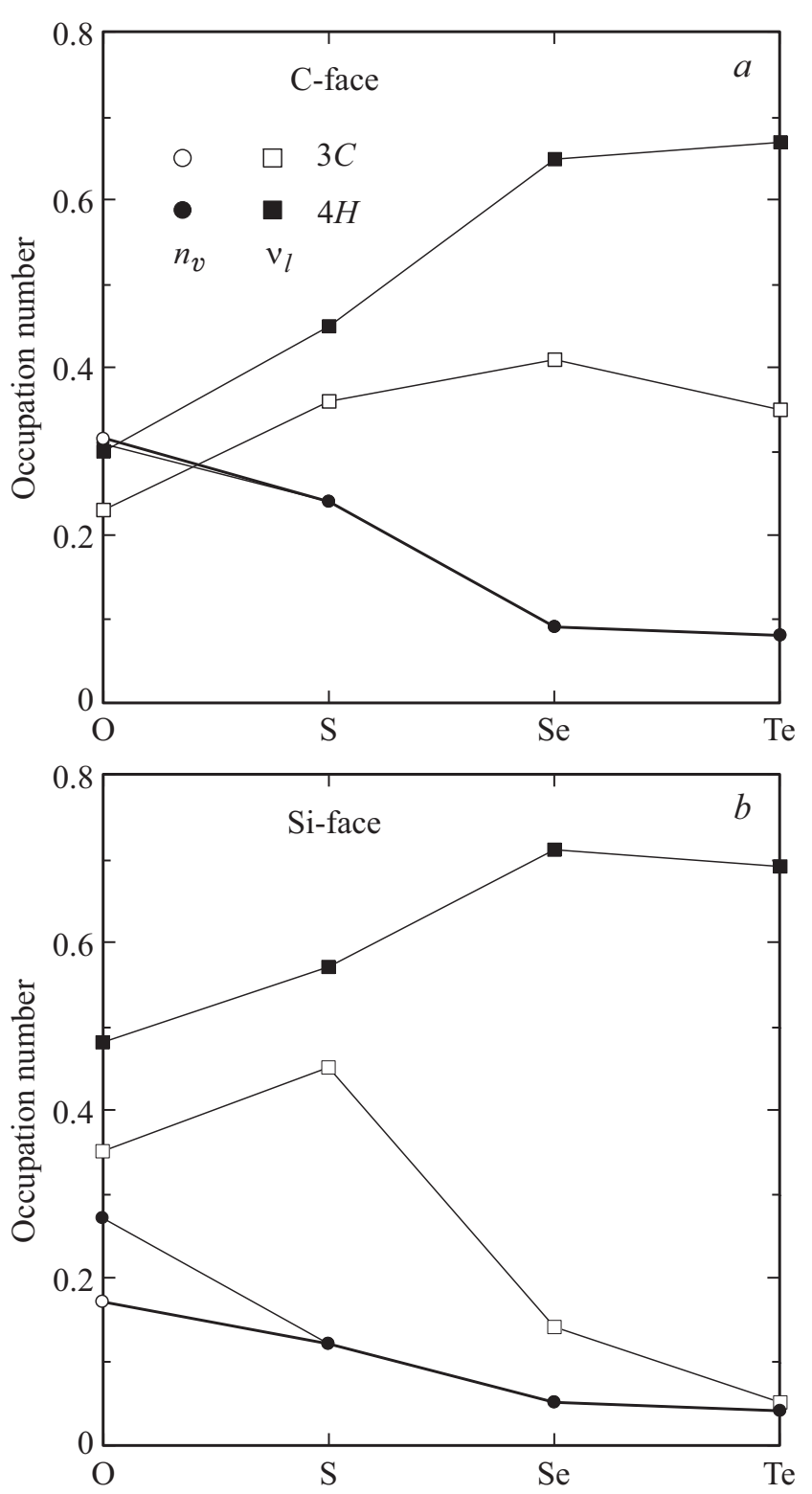

Рис. 2. Адатомы VI группы: числа заполнения зонных $n_{v}$ (кружки) и локальных $v_{l}$ (квадраты) состояний на С- и Si-гранях (соответственно $a$ и $b$ ) политипов $3 C$ (светлые символы) и $4 H$ (темные символы).

в области $|\omega| \leq E_{g} / 2[2,3]$. Число заполнения локального состояния равно

$$
n_{l}=v_{l} \Theta\left(E_{\mathrm{F}}-\omega_{l}\right), \quad v_{l}=\left(1+\frac{\Gamma}{\pi} \frac{E_{g}}{\left(E_{g} / 2\right)^{2}-\omega_{l}^{2}}\right)^{-1}
$$

где $\Theta\left(E_{\mathrm{F}}-\omega_{l}\right)-$ функция Хэвисайда. Значения $\varepsilon_{a}$ и $\omega_{l}$ для адатомов II и IV групп представлены в табл. 3 и 4 , а значения $n_{v}$ и $v_{l}-$ на рис. 1 и 2.

Заряд адатома $Z=1-n$ при адсорбции щелочноземельных металлов и $Z=-n$ при адсорбции атомов подгруппы кислорода. Отметим, что величина $Z$ равна переходу заряда между адатомом и подложкой. В слу- чае адсорбции атомов II группы электрон переходит с адатома на подложку (адатомы являются донорами), для адсорбции атомов VI картина обратная (адатомы являются акцепторами). При определении величины $Z$ большое значение имеет тип проводимости подложки. Если уровень Ферми расположен вблизи дна зоны проводимости (n-тип) и все локальные уровни заполнены $\left(\omega_{l}<E_{\mathrm{F}}\right)$, то получаем минимальный заряд $Z^{<}$для адатомов II группы и максимальный (по модулю) заряд для адатомов VI группы. Если же уровень Ферми лежит вблизи потолка валентной зоны (p-тип) и $\omega_{l}>E_{\mathrm{F}}$, то заряд $Z^{>}$щелочноземельных металлов максимален, а заряд адатомов кислородной подгруппы минимален (по модулю). Значения зарядов $Z^{<}$и $Z^{>}$приведены в табл. 3 и 4.

\section{3. Энергия адсорбции}

Перейдем к оценкам энергии адсорбции $E_{\mathrm{ads}}$, которую можно представить в виде суммы металлической $E_{\text {met }}$ и ионной $E_{\text {ion }}$ составляющих. Металлическую со-

Таблица 3. Переход заряда $Z^{<(>)}$и энергии $E_{\text {ion }}^{<(>)}, E_{\text {ads }}^{<(>)}$ (в эВ) для атомов II группы, адсорбированных на С- и $\mathrm{Si}$-гранях политипов $3 C$ - и $4 H-\mathrm{SiC}$

\begin{tabular}{c|c|c|c|c|c}
\hline $\mathrm{M} / 3 C$-SiC & $\mathrm{Be}$ & $\mathrm{Mg}$ & $\mathrm{Ca}$ & $\mathrm{Sr}$ & $\mathrm{Ba}$ \\
\hline $\mathrm{C}$-грань & & & & & \\
$Z^{<}$ & 0.13 & 0.07 & 0.14 & 0.16 & 0.29 \\
$Z^{>}$ & 0.41 & 0.67 & 0.91 & 0.94 & 0.95 \\
$-E_{\text {ion }}^{<}$ & 0.03 & 0.01 & 0.03 & 0.03 & 0.10 \\
$-E_{\text {ion }}^{>}$ & 0.32 & 0.68 & 1.09 & 1.09 & 1.08 \\
$-E_{\text {ads }}^{<}$ & 1.00 & 0.38 & 0.28 & 0.21 & 0.29 \\
$-E_{\text {ads }}^{>}$ & 0.84 & 1.05 & 1.34 & 1.30 & 1.27 \\
$\mathrm{Si}-$ грань & & & & & \\
$Z^{<}$ & 0.16 & 0 & 0.08 & 0.11 & 0.17 \\
$Z^{>}$ & 0.24 & 0.62 & 0.94 & 0.96 & 0.97 \\
$-E_{\text {ion }}^{<}$ & 0.04 & 0 & 0.01 & 0.01 & 0.03 \\
$-E_{\text {ion }}^{>}$ & 0.09 & 0.50 & 1.01 & 1.00 & 0.99 \\
$-E_{\text {ads }}^{<}$ & 0.58 & 0.23 & 0.24 & 0.21 & 0.22 \\
$-E_{\text {ads }}^{>}$ & 1.12 & 0.73 & 1.24 & 1.20 & 1.18 \\
\hline $\mathrm{M} / 4 H$-SiC & $\mathrm{Be}$ & $\mathrm{Mg}$ & $\mathrm{Ca}$ & $\mathrm{Sr}$ & $\mathrm{Ba}$ \\
\hline $\mathrm{C}$-грань & & & & & \\
$Z^{<}$ & 0.12 & 0.03 & 0.08 & 0.08 & 0.10 \\
$Z^{>}$ & 0.44 & 0.67 & 0.91 & 0.94 & 0.95 \\
$-E_{\text {ion }}^{<}$ & 0.03 & 0 & 0.01 & 0.01 & 0.01 \\
$-E_{\text {ion }}^{>}$ & 0.37 & 0.68 & 1.09 & 1.09 & 1.08 \\
$-E_{\text {ads }}^{<}$ & 0.71 & 0.37 & 0.26 & 0.22 & 0.20 \\
$-E_{\text {ads }}^{>}$ & 1.05 & 1.05 & 1.34 & 1.30 & 1.27 \\
$\mathrm{Si}-г$ гань & & & & & \\
$Z^{<}$ & 0.05 & 0 & 0.04 & 0.05 & 0.06 \\
$Z^{>}$ & 0.68 & 0.62 & 0.91 & 0.96 & 0.97 \\
$-E_{\text {ion }}^{<}$ & 0.04 & 0 & 0 & 0 & 0 \\
$-E_{\text {ion }}^{>}$ & 0.73 & 0.50 & 0.95 & 1.00 & 0.99 \\
$-E_{\text {ads }}^{<}$ & 0.58 & 0.33 & 0.23 & 0.20 & 0.21 \\
$-E_{\text {ads }}^{>}$ & 1.27 & 0.83 & 1.18 & 1.20 & 1.18 \\
& & & & &
\end{tabular}


Таблица 4. То же, что в табл. 3, но для атомов VI группы

\begin{tabular}{c|c|c|c|c}
\hline $\mathrm{X} / 3 C$-SiC & $\mathrm{O}$ & $\mathrm{S}$ & $\mathrm{Se}$ & $\mathrm{Te}$ \\
\hline $\mathrm{C}$-грань & & & & \\
$Z^{<}$ & 0.55 & 0.60 & 0.50 & 0.43 \\
$Z^{>}$ & 0.32 & 0.24 & 0.09 & 0.08 \\
$-E_{\text {ion }}^{<}$ & 0.72 & 0.72 & 0.38 & 0.27 \\
$-E_{\text {ion }}^{>}$ & 0.23 & 0.11 & 0.01 & 0.09 \\
$-E_{\text {ads }}^{<}$ & 1.99 & 1.50 & 0.75 & 0.60 \\
$-E_{\text {ads }}^{>}$ & 1.50 & 0.89 & 0.38 & 0.42 \\
$\mathrm{Si}-$ грань & & & & \\
$Z^{<}$ & 0.52 & 0.57 & 0.19 & 0.09 \\
$Z^{>}$ & 0.17 & 0.12 & 0.05 & 0.04 \\
$-E_{\text {ion }}^{<}$ & 0.51 & 0.65 & 0.05 & 0.01 \\
$-E_{\text {ion }}^{<}$ & 0.05 & 0.03 & 0.04 & 0.02 \\
$-E_{\text {ads }}^{<}$ & 1.78 & 1.43 & 0.42 & 0.34 \\
$-E_{\text {ads }}^{>}$ & 1.32 & 0.81 & 0.41 & 0.35 \\
\hline $\mathrm{X} / 4 H$-SiC & 0 & $\mathrm{~S}$ & $\mathrm{Se}$ & $\mathrm{Te}$ \\
\hline $\mathrm{C}-$-грань & & & & \\
$Z^{<}$ & 0.61 & 0.69 & 0.74 & 0.75 \\
$Z^{>}$ & 0.31 & 0.24 & 0.09 & 0.08 \\
$-E_{\text {ion }}^{<}$ & 0.89 & 0.95 & 0.83 & 0.82 \\
$-E_{\text {ion }}^{>}$ & 0.23 & 0.11 & 0.01 & 0.01 \\
$-E_{\text {ads }}^{<}$ & 2.16 & 1.73 & 1.18 & 1.12 \\
$-E_{\text {ads }}^{>}$ & 1.50 & 0.89 & 0.38 & 0.34 \\
$\mathrm{Si}-$-грань & & & & \\
$Z^{<}$ & 0.75 & 0.69 & 0.76 & 0.73 \\
$Z^{>}$ & 0.27 & 0.12 & 0.05 & 0.04 \\
$-E_{\text {ion }}^{<}$ & 1.05 & 0.77 & 0.75 & 0.67 \\
$-E_{\text {ion }}^{<}$ & 0.14 & 0.02 & 0 & 0 \\
$-E_{\text {ads }}^{<}$ & 1.93 & 1.55 & 1.08 & 0.88 \\
$-E_{\text {ads }}^{>}$ & 1.02 & 0.80 & 0.33 & 0.21 \\
& & & &
\end{tabular}

ставляющую энергии адсорбции $E_{\text {met }}$ оцениваем исходя из соотношения неопределенностей $\Delta x \cdot \Delta p \sim \hbar$, где $\hbar$ - приведенная постоянная Планка. Полагая, что в изолированном атоме $\Delta x \sim r_{a}$, а в адсорбированном состоянии $\Delta x \sim d$, получим выигрыш в кинетической энергии $\Delta E_{\mathrm{kin}} \sim\left(\hbar^{2} / 2 m d^{2}\right)\left[1-\left(r_{a} / d\right)^{2}\right]$, где $m-$ масса свободного электрона. Это понижение в кинетической энергии, связанное с делокализацией электрона при адсорбции, принимаем за оценку металлической составляющей энергии адсорбции:

$$
E_{\mathrm{met}}=-\frac{\hbar^{2}}{2 m d^{2}}\left(1-\frac{r_{a}^{2}}{d^{2}}\right)
$$

Значения $E_{\text {met }}$ для адатомов II и VI групп представлены на рис. 3.

Ионный вклад в энергию адсорбции может быть оценен исходя из электростатической формулы

$$
E_{\text {ion }}=-\frac{(Z e)^{2}}{4 d} .
$$

Значения энергии адсорбции $E_{\mathrm{ads}}^{<(>)}=E_{\mathrm{met}}+E_{\mathrm{ion}}^{<(>)}$представлены в табл. 3 и 4.

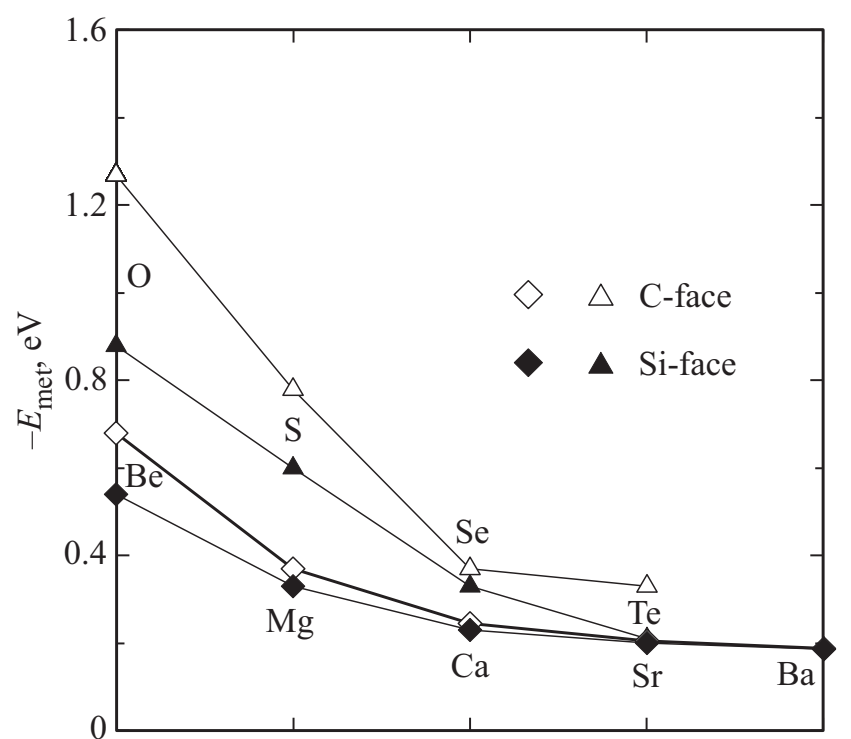

Рис. 3. Металлическая составляющая $E_{\text {met }}$ энергии адсорбции (в эВ). Светлые (темные) символы относятся к адсорбции атомов II (ромбы) и VI (треугольники) групп на $\mathrm{C}(\mathrm{Si})$-грани политипов $\mathrm{SiC}$.

\section{3. Обсуждение результатов}

Прежде всего отметим, что в рядах Ве $\rightarrow$ Ва и $\mathrm{O} \rightarrow \mathrm{Te}$ (за исключением $\mathrm{S}$ ) имеет место рост значений $\varepsilon_{a}$ (см. табл. 1 и 2). В случае щелочноземельных металлов эта тенденция объясняется уменьшением потенциала ионизации $I$ при переходе от бериллия к барию [6]. Рост $\varepsilon_{a}$ в ряду подгруппы кислорода связан с увеличением электронного сродства $A$ и убылью кулоновского сдвига $e^{2} / 4 d$. Тот же характер изменений имеет место для $\omega_{l}$ (см. табл. 1 и 2), так как $\omega_{l} \propto \varepsilon_{a}$ [2].

Изменения чисел заполнения носят более сложный характер. Из выражения (2) следует, что $n_{v}$ уменьшается с ростом $\varepsilon_{a}$. Это приводит к монотонному уменьшению значений $n_{v}$ в рядах $\mathrm{Be} \rightarrow \mathrm{Ba} \mathrm{и} \mathrm{O} \rightarrow$ Те (рис. 1). С другой стороны, из выражения (4) вытекает, что зависимость $n_{l}\left(\omega_{l}\right)$ является немонотонной: $n_{l}$ достигает максимума при $\omega_{l}=0$ и стремятся к нулю при $\omega_{l} \rightarrow \pm E_{g} / 2$. Поэтому немонотонным является изменение $n_{l}$ в рядах $\mathrm{Be} \rightarrow \mathrm{Ba} \mathrm{и} \mathrm{O} \rightarrow$ Те (рис. 1$)$.

Перейдем к зарядам адатомов. Из табл. 3 следует, что у адатомов II группы заряды положительны. В случае атомов, адсорбированных на С-грани $3 C$-политипа, заряды $Z^{<(>)}$возрастают в ряду $\mathrm{Be} \rightarrow \mathrm{Ba}$ (исключением является заряд магния $\left.Z^{<} \ll 1\right)$. То же относится к зарядам $Z^{>}$при адсорбции на $\mathrm{Si}$-грани. В случае $4 H$-политипа монотонно возрастают только заряды $Z^{>}$ (исключением является заряд магния для случая адсорбции на $\mathrm{Si}$-грани). Во всех случаях имеем $Z^{>}>Z^{<}$.

Из табл. 4 следует, что у адатомов VI группы заряды отрицательны. В случае адсорбции на С-грани $3 C$-политипа значения $\left|Z^{<(>)}\right|$убывают в ряду $\mathrm{O} \rightarrow \mathrm{Te}$ (исключением являются адатомы серы). Та же зави- 
симость характерна для $\left|Z^{>}\right|$при адсорбции на Cи $\mathrm{Si-гранях} 4 H$-политипа. Во всех случаях имеем $\left|Z^{<}\right|>\left|Z^{>}\right|$.

Обратимся теперь к оценкам энергии адсорбции $E_{\mathrm{ads}}^{<(>)}$. Рис. 3 демонстрирует спад значений металлического вклада $\left|E_{\text {met }}\right|$ в рядах $\mathrm{Be} \rightarrow \mathrm{Ba} \mathrm{и} \mathrm{O} \rightarrow$ Те. Эта тенденция связана с изменением длин адсорбционных связей $d$, которые растут при переходе от первого элемента ряда к последнему (см. табл. 1 и 2). Для адатомов VI группы величины $\left|E_{\text {met }}\right|$ выше, чем для адатомов II группы. То же неравенство имеет место для адсорбции на C- и Si-гранях. Переходя к ионным составляющим энергии адсорбции, следует подчеркнуть, что для адатомов II группы значения $\left|E_{\text {ion }}^{<}\right|$не превосходят 0.1 эВ, так что $\left|E_{\text {ion }}^{>}\right| \gg\left|E_{\text {ion }}^{<}\right|$. Для адатомов VI группы имеем $\left|E_{\text {ion }}^{>}\right|<\left|E_{\text {ion }}^{<}\right|$. Значения $\left|E_{\text {ion }}^{<(>)}\right|$в ряду $\mathrm{O} \rightarrow$ Те убывают (исключения составляют $\left|E_{\text {ion }}^{<}\right|$для атома серы и $\left|E_{\text {ion }}^{>}\right|$ для атома селена).

Результаты оценок суммарной энергии адсорбции $E_{\text {ads }}^{<(>)}$приведены в табл. 3 и 4. Сравнение с результатами работы [2] показывает, что характер изменения энергии адсорбции в рядах $\mathrm{Be} \rightarrow \mathrm{Ba}, \mathrm{O} \rightarrow \mathrm{Te}$ и $\mathrm{Li} \rightarrow \mathrm{Cs}$, $\mathrm{F} \rightarrow \mathrm{I}$ одинаков. В целом значения $\left|E_{\mathrm{ads}}^{<(>)}\right|$для адатомов II и VI групп несколько ниже, чем для адатомов I и VII групп. К сожалению, полученные нами здесь оценки сравнить практически не с чем. Действительно, мы отыскали только работу [8], где из первых принципов рассчитывалась энергия связи $E_{b}=-E_{\text {ads }}$ атомарного кислорода с $\mathrm{Si}$-гранью кубического $\mathrm{SiC}$ и было получено значение $E_{b} \approx 3$ эВ, что в $1.5-2$ раза превышает нашу оценку.

\section{4. Заключение}

Итак, для оценок адсорбционных характеристик политипов карбида кремния мы применяем одну и ту же модель к адатомам I, II, VI и VII групп. При этом игнорируются различия между атомами I (VII) группы, с одной стороны, и атомами II (VI) группы - с другой, состоящие в том, что в первом случае внешняя электронная оболочка атома содержит один $s$-электрон (одну $p$-дырку), тогда как во втором случае имеются два $s$-электрона (две $p$-дырки). Допустимость применения одночастичного подхода к описанию адсорбции атомов с валентными электронами, находящимися в состояниях $s^{2}$ и $p^{4}$, продемонстрирована в работах $[9,10]$. Таким образом, в задаче об адсорбции мы рассматриваем лишь один электрон, участвующий в переходе между адатомом и подложкой, игнорируя электроны внутренних оболочек.

Вновь подчеркнем, что предлагаемый подход предназначен для выявления тенденций изменения перехода заряда и энергии адсорбции при переходе от адатома к адатому. Воспользовавшись полученными здесь и в [2] результатами, легко оценить также изменения работы выхода $[3,4]$ и поверхностной проводимости [11] адсорбционной системы.

\section{Конфликт интересов}

Авторы заявляют, что у них нет конфликта интересов.

\section{Список литературы}

[1] А.А. Лебедев, П.А. Иванов, М.Е. Левинштейн, Е.Н. Мохов, С.С. Нагалюк, А.Н. Анисимов, П.Г. Баранов. УФН, 189, 803 (2019).

[2] С.Ю. Давыдов, О.В. Посредник. ФТП, 54, 1197 (2020).

[3] С.Ю. Давыдов. Теория адсорбции: метод модельных гамильтонианов (СПб., изд-во СПбГЭТУ „ЛЭТИ“, 2013). twirpx.com/file/1596114/

[4] С.Ю. Давыдов, А.А. Лебедев, О.В. Посредник. Элементарное введение в теорию наносистем (СПб., Лань, 2014).

[5] С.Ю. Давыдов. ФТП, 53, 706 (2019).

[6] Физические величины. Справочник, под ред. Е.С. Григорьева, Е.3. Мейлихова (М., Энергоатомиздат, 1991).

[7] C.Ю. Давыдов, С.В. Трошин. ФТТ, 49, 1508 (2007).

[8] R. Rurali, E. Wachowich, P. Ordeon, P. Godingnon, J. Rebollo, P. Hildegaard. Mater. Sci. Forum, 457-460, 1293 (2004).

[9] С.Ю. Давыдов, А.В. Павлык. Письма ЖТФ, 29 (12), 33 (2003).

[10] С.Ю. Давыдов. ФТТ, 47, 1711 (2005).

[11] Д.Г. Аньчков, С.Ю. Давыдов, С.В. Трошин. Письма ЖТФ, 33 (18), 47 (2007).

Редактор Г.А. Оганесян

\section{Adsorption of II and VI groups atoms on the silicon carbide polytypes}

\author{
S.Yu. Davydov' ${ }^{1}$ O.V. Posrednik ${ }^{2}$ \\ ${ }^{1}$ loffe Institute, \\ 194021 St. Petersburg, Russia \\ ${ }^{2}$ St. Petersburg State Electrotechnical University (ETU), \\ 197376 St. Petersburg, Russia
}

Abstract Within the scope of the Haldane-Anderson model the estimates of charge transfer and adsorption energy for alkali metals and halogens atoms on the C- and Si-faces of $3 \mathrm{C}-, 6 \mathrm{H}$ - and $4 \mathrm{H}$-SiC polytypes are obtained. Band and local states contributions to the formation of adatom's charge and ionic and metallic parts of the adsorption energy are clarified. 